Apidologie, 1983, 14 (3), 183-190.

\title{
ÜBER DEN EINFLUSS BLUMENHAFTER DÜFTE AUF DAS DUFTMARKIEREN DER SAMMELBIENE
}

\author{
Karl Theodor WILHELM und Walter Wilhelm PFLUMM
}

Fachbereich Biologie der Universität, Erwin-Schrödinger-Straße, D-6750 Kaiserslautern

\section{ZUSAMMENFASSUNG}

An der Futterquelle vorhandener Duft von Maiglöckchenöl, Rosenöl oder Geraniol hat bei Nektarsammlerinnen (Apis mellifica) keinen Einfluß auf die Dauer des Duftmarkierens während des Saugens.

Nektarsammlerinnen kennzeichnen gute Futterplätze mit dem Pheromon der Nassanoffschen Drüse (v. FrISCH, 1923, 1935). Folgende Faktoren beeinflussen die Dauer dieses Duftmarkierens an einer Zuckerwasser spendenden Futterquelle : a) die Konzentration der Lösung (Pflumm, 1969; Pflumm et al., 1978); b) deren Zuflußrate (früher als Zuflußgeschwindigkeit bezeichnet : PfLUMM, 1969; NúNEZ, 1971); c) die Trachtverhältnisse (Pflumm et al., 1978). Erhöht oder erniedrigt man die Konzentration des Sterzeldufts an der Futterquelle oder bietet man dessen wirksamste Komponente Citral (Butler und CALAM, 1969), wirkt sich dies auf die Dauer des Duftmarkierens nicht aus - d.h. es findet keine olfaktorische Rückkopplung statt (Pflumm und Wilhelm, 1982).

Ob blumenhafte Düfte das Betätigen des Duftorgans an künstlichen Futterquellen beeinflussen, wurde bisher nicht geprüft. An - mit Zuckerwasser versehenen - Blüten soll nach FREE (1968) Duftmarkieren nur dann stattfinden, wenn diese duftlos sind.

Im folgenden sind Experimente zum Einflu $\beta$ von Blumendüften geschildert. Außer etherischen Ölen verwendeten wir Geraniol, welches nicht nur den mengenmäßig größten Anteil des Sekrets der Nassanoffschen Drüse ausmacht (BOCH und SHEARER, 1962), sondern auch in Blütendüften vorkommt (PAECH, 1950). 
Da " die Bedingungen im Flugraum... nicht das Niveau der Haltung im Freien » erreichen (RUTTNER und KoENIGER, 1977), haben wir im Freiland einige der früher in einem Flugraum durchgeführten Versuche zur olfaktorischen Rückkopplung wiederholt.

\section{METHODIK}

Die Experimente führten wir von Juni bis Oktober 1982 durch. Die Sammlerinnen wurden an Blüten weggefangen, auf dem Thorax mit einem Farbtupfen markiert und an eine künstliche Futterquelle (Porzellanschale, $\varnothing 12 \mathrm{~cm}$ ) gesetzt. Nach der Andressur verkehrten die Versuchsbienen nur noch an dieser Futterquelle. Sie stand beschattet $75 \mathrm{~m}$ vom Bienenstand entfernt. Jede Biene konnte trinken, so schnell sie vermochte, d.h. es herrschte "volier Zufluß » der Lösung.

Wir verwendeten handelsüblichen Rohrzucker (Konzentrationsangaben in den Abbildungen; $\%=g$ pro $100 \mathrm{~g}$ ). Pro Tag boten wir nur eine Lösung bestimmter Konzentration.

Gemessen wurde die Dauer des Duftmarkierens während des Saugens (zur Nomenklatur vgl. Pflumm, 1969). Gelegentlich hielt eine Sammlerin das Duftorgan bis zum Abflug ausgestülpt.

Die Luftemperatur folgte dem Tagesgang. Da mit einem - bisher nicht untersuchten - Einfluß dieser Größe auf das Duftmarkieren zu rechnen ist, werteten wir nur solche Versuchsergebnisse aus, die bei etwa gleicher Temperatur gewonnen worden waren (Temperaturangaben in Abb. 1 und Legende Abb. 2).

Wir prüften Rosen- und Maiglöckchenöl (aus der Parfümindustrie) sowie Geraniol (Merck, Darmstadt). Pro Tag wurde nur eine Substanz getestet. Diese gaben wir in einen Glaszylinder (Volumen $16 \mathrm{ml}$ ), welchen wir zu Saugbeginn über die Biene stülpten. Die Menge dosierten wir so, daß die Biene nicht gestört war (s. hierzu Pflumm und Wilhelm, 1982). Besuche mit geänderter olfaktorischer Reizsituation und Kontrollbesuche wechselten in statistischer Zufallsreihenfolge (Beispiel einer solchen in Abb. 1).

Bei der Auswertung errechneten wir aus den in einer bestimmten Situation erhaltenen Meßwerten den Zentralwert. Für die Darstellung der Abb. 2 bezogen wir jeweils den bei Besuchen mit Duftstoff erhaltenen Zentralwert auf den Zentralwert der unbeeinflußten Besuche ( $=$ Kontrolle). Dies war erforderlich, weil einerseits die Experimente zu verschiedenen Jahreszeiten durchgeführt worden waren, andererseits große interindividuelle Unterschiede auftraten (Literaturangaben hierzu bei PFLUMM et al., 1978). In die statistische Bearbeitung der Daten gingen die nicht normierten Werte ein. Wir verwendeten den U-Test bei zweiseitiger Fragestellung. Als Signifikanzniveau wählten wir $5 \%$.

\section{ERGEBNISSE}

Die Dauer des Duftmarkierens während des Saugens bei unbeeinflußten Besuchen unterscheidet sich nicht signifikant von der bei Besuchen, während derer die Biene beim Saugen durch Maiglöckchenduft, Rosenduft oder Geraniol olfaktorisch beeinflußt wird (Abb. 1 und 2).

Wir erhielten bei allen untersuchten Bienen Resultate, welche den in Abb. 2 dargestellten entsprechen; wir stellten in dieser Abbildung aber nur solche Versuchsergebnisse dar, die jeweils bei etwa derselben Temperatur und zur gleichen Jahreszeit gewonnen worden waren. 


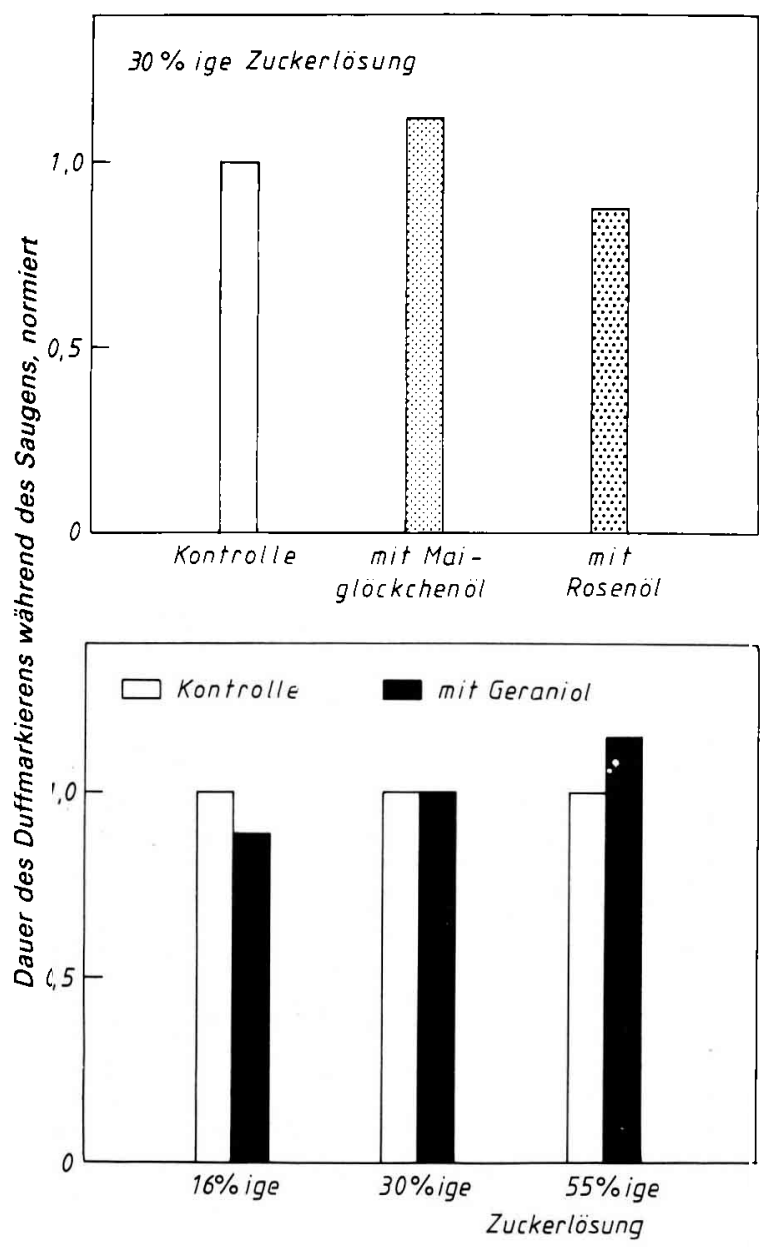

Aвв. 2. - Einfluß verschiedener Dufistoffe an der Futterquelle auf die Dauer des Duftmarkierens während des Saugens.

Zentralwerte. 5 Bienen. Anzahl der Versuche pro Biene : $7<\mathrm{n}<41$. Oben : Die linke Säule stellt sämtliche zur mittleren und rechten Säule gehörenden Kontrollwerte dar. Mittlere Säule : Oktober; Temperatur $15 \circ \mathrm{C}$. Rechte Säule : September; Temperatur $26 \pm 1 \circ \mathrm{C}$. Unten : Linkes Säulenpaar : Juli; Temperatur $29 \pm 1$ oC. Mittleres Säulenpaar : September; Temperatur $26 \pm 1$ oC. Rechtes Säulenpaar : Juli; Temperatur $29 \pm 1$ oC. Unterschied zwischen dem Wert der Kontrolle und dem Wert für den Duftstoff jeweils nicht signifikant.

FIG. 2. - Influence of different odorous substances at the food source on the duration of scent marking behaviour during drinking

Medians. 5 bees. Number of trials per bee : $7<\mathrm{n}<41$.

Above : trials with $30 \%$ sugar solution. The left column shows control measurements for the middle and right column. Middle column : lily of the valley oil, October, temperature $15{ }^{\circ} \mathrm{C}$.

Right column : rose oil, September, temperature $26 \pm 1{ }^{\circ} \mathrm{C}$.

Below :trials with Geraniol and different concentrations of sugar solution. Left column : $16 \%$ sugar solution, July, temperature $29 \pm 1$ oC. Middle column : $30 \%$ sugar solution, September, temperature $26 \pm 1 \circ \mathrm{C}$. Right column : $55 \%$ sugar solution, July, temperature $29 \pm 1{ }^{\circ} \mathrm{C}$.

Differences between values and control not significant. 
Ein möglicher Einfluß von Duftstoff (geprüft wurde Geraniol) auf die Dauer des Ausstülpens der Nassanoffschen Drüse läßt sich auch dann nicht nachweisen, wenn statt einer $30 \%$ igen eine $16 \%$ ige oder $55 \%$ ige Zuckerlösung geboten wird (Abb. 2, unten).

Erhöht man im Freiland an der Futterquelle die Konzentration des Sterzeldufts, indem man ein Gläschen über die saugende und duftmarkierende Biene stülpt, ändert diese ihr Duftmarkierverhalten nicht. Diese Ergebnisse sind in Abb. 2 nicht dargestellt, sie bestätigen unsere in einem Flugraum gewonnenen Resultate (PFLumM und WILHELM, 1982).

\section{DISKUSSION}

Nach den Ergebnissen der vorliegenden Arbeit haben an der Futterquelle vorhandene Blütendüfte (Duftstoffgemische) keinen Einfluß auf die Dauer des Duftmarkierens. Dasselbe gilt nach Abb. 1 und 2 für reines Geraniol. Dieses bildet den Hauptbestandteil dẹs Sterzeldufts (BoCH und ShEarer, 1962). Man kann einer Sammlerin mit Geraniol allerdings keinen Sterzelduft "vortäuschen ", da sie zwischen beiden Duftstoffen einwandfrei unterscheiden kann (VARESCHI und KAISSLING, 1970). Geraniol ist auch sehr häufig in etherischen Ölen enthalten - so im Rosenöl bis zu $75 \%$ (PAECH, 1950).

Unsere Befunde stehen im Gegensatz zu Aussagen von FreE (1968). Nach diesem Autor soll sowohl Blumenduft als auch Geraniol das Ausstülpen der Nassanoffschen Drüse beeinflussen. FreE ließ Zuckerlösung bestimmter Konzentration einerseits aus Rosen (im Juni), andererseits aus Sonnenblumen (im September) sammeln. An Rosen war der Anteil duftmarkierender Bienen niedriger als der Anteil bei den an Sonnenblumen sammelnden. Bei diesen Experimenten lagen nicht nur verschiedene olfaktorische Situationen an der Futterquelle vor, die Trachtverhältnisse waren ebenfalls unterschiedlich. Die Ergebnisse der vorliegenden Arbeit bestätigen eine frühere Vermutung (PFLUMm, 1970); danach sind die von FrEE an Blumen beobachteten Unterschiede im. Duftmarkierverhalten auf die verschiedenen Trachtverhältnisse zurückzuführen. Die bei Versuchen mit Geraniol erhaltenen Resultate FrEE (an den Kontrollschalen war der Anteil duftmarkierender Bienen höher als an den Schalen mit Geraniol) dürften dadurch zustandegekommen sein, daß FREE Geraniol in zu hoher Konzentration geboten und so der Futterquelle eine « abstoßende » Wirkung verliehen hatte. Durch zu hohe Duftstoffkonzentration gestörte Bienen unterbrechen nämlich das Saugen und ziehen das Duftorgan ein; beim erneuten Saugbeginn stülpen sie es nicht wieder aus (vgl. auch die Ausführungen bei Shearer und Boch, 1966 sowie Pflumm und Wilhelm, 1982). 
Wir erbringen hiermit einen weiteren Beweis dafür, daß die Dauer des Duftmarkierens an der Futterquelle nur von der Konzentration und Zuflußrate der Zuckerlösung sowie den Trachtverhältnissen gesteuert wird (PFLumM, 1969, 1970; Núñez, 1971 ; Pflumm et al., 1978; Pflumm und Wilhelm, 1982). Eine weitere - das Verhalten der Nektarsammlerin am Futterplatz stark beeinflussende - Größe ist die Lufttemperatur an der Futterquelle. Versuche zur Abhängigkeit des Duftmarkierens von der Temperatur sind im Gange.

Eingegangen im Mai 1983. Reçu pour publication en mai 1983.

\title{
DANK
}

Wir danken den Herren J. WeIHRAUCH und T. Moster für tatkräftige Hilfe bei der Versuchsdurchführung, Herrn Dr. D. Graham für die Korrektur der englischen Zusammenfassung.

\author{
RÉSUMÉ \\ INFLUENCE D'ODEURS FLORALES SUR LE COMPORTEMENT \\ DE MARQUAGE OLFACTIF DES ABEILLES BUTINEUSES
}

On sait que la durée du comportement de marquage olfactif (exposition de la glande de Nasonov) par les butineuses de nectar à la source de nourriture n'est pas influencée par la présence de l'odeur de la glande ou de son composé, le citral (Pflumm et Wilhelm, 1982). Ce travail examine l'hypothèse de FreE (1968), selon laquelle des odeurs florales ou du géraniol influent sur la durée d'exposition de la glande de Nasonov.

Lorsque l'abeille commence à boire, on retourne sur elle un tube en verre de $16 \mathrm{ml}$ contenant une gouttelette d'essence de muguet, de rose ou de géraniol. On mesure le temps pendant lequel l'organe olfactif émetteur est sorti au cours de la prise de nourriture. Les odeurs florales (huiles essentielles) ni le géraniol n'ont d'influence sur cette durée (Fig. 1 et 2). L'odeur (c'est le géraniol qui est testé) n'exerce aucune action sur la durée du marquage olfactif, lorsqu'on donne une solution de saccharose à 16 ou $55 \%$ à la place de celle à $30 \%$ (Fig. 2).

Les résultats de FrEE (1968) ne doivent pas être attribués aux diverses odeurs florales à la source de nourriture, mais plutôt aux différences dans l'état d'approvisionnement général de la ruche en nourriture. Dans ses expériences avec le géraniol, FrEE ajoutait la substance odorante à une concentration bien trop élevée : des abeilles perturbées rentraient leur organe de Nasonov.

Nos résultats fournissent une nouvelle preuve que l'abeille règle la durée du marquage olfactif à la source de nourriture uniquement d'après la concentration et le débit d'alimentation de solution sucrée et l'état d'approvisionnement général de la ruche en nourriture (Pflumm, 1969, 1970; NúÑEZ, 1971; PFLUMm et al., 1978; Pflumm et Wilhelm, 1982). 


\section{SUMMARY}

\section{INFLUENCE OF FLOWERLIKE FRAGRANCES AT THE FOOD SOURCE ON THE SCENT MARKING BEHAVIOUR OF FORAGING HONEYBEES (APIS MELLIFICA)}

It is now known, that the duration of the scent marking behaviour (exposure of the Nasonov gland) of nectar gathering honeybees (Apis mellifica) at the food source is not influenced by the presence of Nasonov scent or its component citral (PFLUMm and WILHELM, 1982). This paper investigates the assumption made by FREE (1968) that flowerlike fragrances or geraniol affect the exposure of the Nasonov gland.

As the bee began to drink, we inverted a $16 \mathrm{ml}$ glass cylinder over the bees containing a droplet of oil from wood lily, rose oil or geraniol. We measured the time the bee spent with its scent organ everted during drinking. Flowerlike fragrances (ethereal oils) or geraniol did not influence this duration (Figs. 1 and 2). We offered sucrose solution of 16,30 or $55 \%$ and found that scent (we tested geraniol) had no influence on the duration of scent marking behaviour (Fig. 2).

The results obtained by FrEE (1968) must not be ascribed to the different flower fragrances at the food source but rather to the differences in the state of the hive's general food supply. In FREE's experiments with geraniol, the concentration of the odourous substance was much too high : disturbed bees withdraw their scent organ.

Our results provide further evidence that the bee controls the duration of her scent marking behaviour at the food source only according to the concentration and rate of supply of the sugar solution and the state of the hive's general food supply (PFLumm, 1969, 1970; NÚnez, 1971; Pflumm et al., 1978; Pflumm and WILHELM, 1982).

\section{LITERATUR}

BOCH R. und SHEARER D.A., 1962. - Identification of geraniol as the active component in the Nassanoff pheromone of the honey bee. Nature, 194, 704-706.

Butler C.G. und CALAM D.H., 1969. - Pheromones of the honey bee - the secretion of the Nassanoff gland of the worker. J. Insect Physiol., 15, 237-244.

FREE J. B., 1968. - The conditions under which foraging honeybees expose their Nasonov gland. J. apic. Res., 7, 139-145.

Frisch K. v., 1923. - Über die « Sprache » der Bienen, eine tierpsychologische Untersuchung. Zool. Jb., Abt. allg. Zool. u. Physiol., 40, 1-186.

Frisch K. v., 1935. - Über den Geschmackssinn der Bienen. Z. vergl. Physiol., 21, 1-156.

NúNEZ J.A., 1971. - Beobachtungen an sozialbezogenen Verhaltensweisen von Sammelbienen. Z. Tierpsychol., 28, 1-18.

PAECH K., 1950. - Biochemie und Physiologie der sekundären Pflanzenstoffe. Springer, Berlin Göttingen - Heidelberg.

Pflum W., 1969. -- Beziehungen zwischen Putzverhalten und Sammelbereitschaft bei der Honigbiene. Z. vergl. Physiol., 64, 1-36.

Pflumm W., 1970. - Auslösende Faktoren beim Duftmarkieren von Sammelbienen. Experientia, 26, 318-319.

Prulmm W., Peschke Cornelia, Wilhelm K. und Cruse H., 1978. - Einfluß der - in einem Flugraum kontrollierten - Trachtverhältnisse auf das Duftmarkieren und die Abflugmagenfüllung der Sammelbiene. Apidologie, 9, 349-362.

Pflum W. und Wilhelm K., 1982. - Olfactory feedback in the scent marking behaviour of foraging honeybees at the food source? Physiol. Entomol. 7, 203-207. 
RuttNer F. und Koeniger N. (redig.) 1977. - Bienenhaltung in Flugräumen; Symposium über Flugraumtechnik. Insectes sociaux, 24, 279-302.

SheARER D.A. und BOCH R., 1966. - Citral in the Nassanoff pheromone of the honey bee. J. Insect Physiol., 12, 1513-1521.

VAReschI E. und Kalssling K.-E., 1970. - Dressur von Bienenarbeiterinnen und Drohnen auf Pheromone und andere Duftstoffe. Z. vergl. Physiol., 66, 22-26. 